\title{
A REVIEW ON THE FABRICATION OF POLYURETHANE NANOFIBERS AND ITS POTENTIAL APPLICATIONS
}

\author{
Dinesh Shah*
}

\begin{abstract}
The electrospinning process is highlighted with the ability of fabricating fibers with diameters on the nanometer scale, small inter-fibrous pore size and high porosity, vast possibilities for functionalization with high surface area to volume or mass ratio, ease of use and instrument setup, and adaptability. It attracted a great deal of attention due to its unique properties. Different polymers have been successfully electrospun into ultrafine fibers using this technique including synthetic polymers such as polyurethane (PU). Electrospun PU nanofiber mats exhibiting good mechanical properties may have a wide variety of potential applications in high-performance air filters, protective textiles, wound dressing materials, sensors, drug delivery, etc.
\end{abstract}

Key words: Electrospinning, polyurethane, nanofibers, applications.

\section{INTRODUCTION}

Electrospinning is a powerful technique to produce polymer nanofibers with a diameter ranging from micro to nanometer scale, depending upon the solution properties (viscosity, conductivity and surface tension)and processing variables (flow-rate,applied voltage, and working distance (Ramakrishna, Fujihara, Teo, Lim \& Ma, 2005). Melt electrospinning produces fat fibers due to the low conductivity and high viscosity of polymer melt in nature, whereas solution electrospinning yields thin fibers by carefully controlling the solution properties and has been successfully applied to numerous polymers (Ramakrishna, Fujihara, Teo, Lim \& Ma, 2005, Huang, Zhang, Kotaki \& Ramakrishna 2003). It is well documented that viscosity is the most important solution property in determining the fiber diameter; the lower the solution viscosity, the thinner the fibers. Technically, almost any soluble polymer with a sufficiently high molecular weight can be electrospun, and various polymers have been

* Mr. Shah is an Assistant Professor, Institute of Engineering, Thapathali Campus,Thapathali, TU. 


\section{0}

A REVIEW ON THE FABRICATION OF POLYURETHANE ...

successfully electrospun into nanofibers cost-effectively compared to the other methods (Sen, 2004).

The method is applied successfully electrospun different polymers into ultrafine fibers including synthetic polymers such as PU. PUs contains urethane group $(-\mathrm{NH}-(\mathrm{C}=\mathrm{O})-\mathrm{O}-)$ in common, while most PUs are thermosetting polymers, in contrast to thermosetting polymers, thermoplastic polyurethanes (TPUs) melt when they are heated and are easy to use in manufacturing processes. Varying the structure of PUs, their properties can be varied in a wide range. PUs are formed by reaction of polyisocyanates with hydroxyl-containing compounds (Demir, 2002). Desired properties can be tailored by selecting the type of isocyanate and polyols, or combination of isocyanates and combination of polyols. Strong intermolecular bonds make polyurethanes useful for diverse applications in adhesives and coatings, also in elastomers, foams, and medical applications because of their good biocompatibility (Khil, 2003). The factors determining properties of a polyurethane elastomer are: structure of the polyol, type of diisocyanate, type of the chain extender, molar ratio $\mathrm{NCO} / \mathrm{OH}$, soft-segment concentration, molecular weight of the polyol and filler. In polyurethane elastomers, chains are linear, and cross-linking was achieved by physical bonds and hard domain formation. They flow when they are melted and harden by cooling (thermoplastic behavior (Barick \& Tripathy 2010)...Displaying reversible cross-linking, domains are destroyed above the melting point of the hard phase, but are reformed when they get cooled. These materials are called "thermoplastic urethanes" (TPUs). Linear polymers have good impact strength, good physical properties, and excellent processibility, but limited thermal stability. On the other hand, thermoset polymers have higher thermal stability, but sometimes lower impact strength.

\section{MATERIALS AND METHOD}

The vast selection of polyols, isocyanates, and chain extenders allows PUs to be varied from soft thermoplastic elastomers to adhesives, coatings, flexible foams, and rigid thermosets. PU is often chosen as a material for composing a nanoweb due to its chemical stability, mass transport, good mechanical properties, and also excellent nanofiber forming characteristic (Padois, 2010). It has reported that semipermeable dressings, many of which are PU, enhance wound healing. Degradable and biocompatible aliphatic PU could also be formed into scaffolds via melt electrospinning 
(Martin, 2012). Varying the structure of PUs, the properties of PUs can vary in a wide range. The flexibility to tailor the structure during processing is one of the main advantages of PUs over other types of polymers. A lot of different types of PU used in electrospinning process, some of them were synthesized before electrospinning according to the researchers intended use and some of them were used as they received (Mi H-Y, 2013).

There are many parameters that affect the electrospinning process and the resultant fiber morphology. Several authors investigated the effects of solution and process parameters such as material composition, concentration, rheological properties, applied voltage, tip-to collector distance, collector types on the resultant PU nanofibers. Among these parameters, polymer solution properties have the most significant influences on the process and the resultant fiber morphology, since viscoelastic and gravitational forces play a major role[9]. Viscoelastic force depends on polymer solution concentration, average molecular weight of the polymer, final viscosity, and surface tension of the solution.

\section{RESULT AND ANALYSIS}

As Electrospinning solution properties are also directly related to the solvent type, hence the type of solvent and their concentration influenced the morphology of electrospun nanofiber mat significantly. Most commonly used solvents that dissolve PUs are highly polar organic solvents such as N,N-dimethylformamide (DMF), dimethylsulfoxide (DMSO), N-Methylpyrrolidone and tetrahydrofuran (THF).It is found that the morphology of the resultant nanofibers changed significantly with the solvent selection.

The effects of the incorporation of ethyl acetate or tetrahydrofuran into TPU/DMF solvent system were investigated. The solutions of TPU in pure DMF and DMF/EA were found to be easily electrospinnable. DMF seemed to be the best solvent to dissolve TPU pellets but diluting TPU solutions with 10 or 20 percent of EA (Ethyl Acetate) ensured positive effect on fiber diameter by means of achieving thinner fibers (Yanilmaz, Kalaoglu \& Karakas 2013). Incorporation of THF to DMF led to thicker fibers compared to TPU/DMF solutions. With the increasing THF volume fraction, electrospinning is restricted due to high viscosity and low conductivity. The diameter of the nanofibers produced by electrospinning is a key parameter for most of the applications. The diameter of the nanofibers defines the structural features such as pore sizes and specific surface areas. 
Solution parameters, especially polymer concentration and the spinning parameters including feeding rate, applied voltage, tip to collector distance, have the strongest impact on the fiber diameter. The properties of PUs can vary in a wide range according to the structure of PU, thus selection of particular parameters is most important for the selected PUs.

The DMF is used for TPU solutions and investigated the effect of TPU concentration $(6,8,10,12$, and 14 percent (w/w) (Mondal 2014) represented in figure 1.given below. This result showed that 6 percent of TPU concentration is too low and 14 percent is too high for smooth nanofibers and as expected nanofiber diameter increased with increasing TPU concentration. With the increasing tip to collector distance, they were able to achieve better nanofiber morphologies and thinner nanofibers. They concluded that in case of thinner and smoother nanofiber production, 10 percent of TPU concentration, $20 \mathrm{~cm}$ tip to collector distance, $0.3 \mathrm{ml} / \mathrm{h}$ feeding rate, and $13 \mathrm{kV}$ applied voltage are suitable (Yanilmaz, Kalaoglu \& Karakas, 2013, Mondal 2014).

Figure 1: (a) 6 percent, (b) 8 percent, (c) 10 percent, (d) 12 percent, and (e) 14 percent $\mathrm{w} / \mathrm{w}$ Electropsun TPU nanofibers.

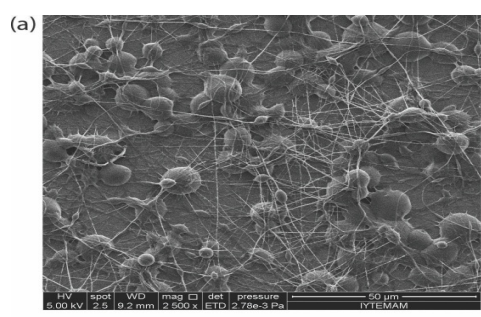

(c)

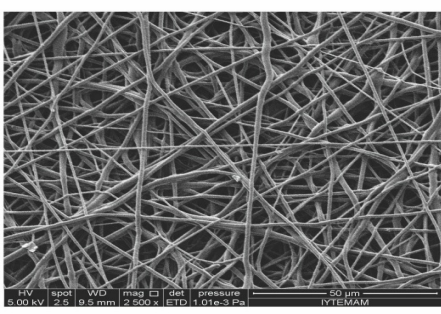

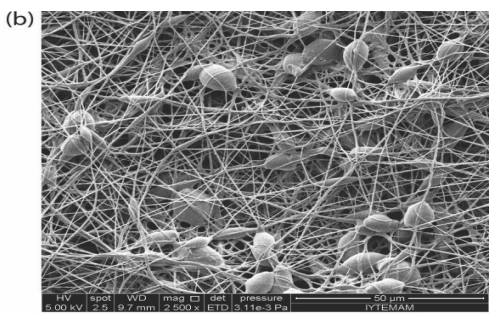

(d)

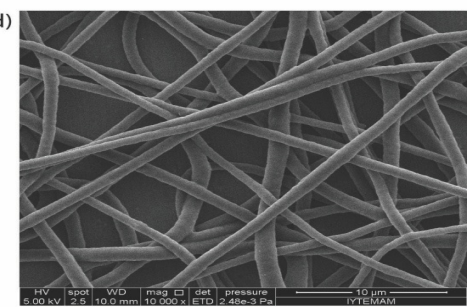

(e)

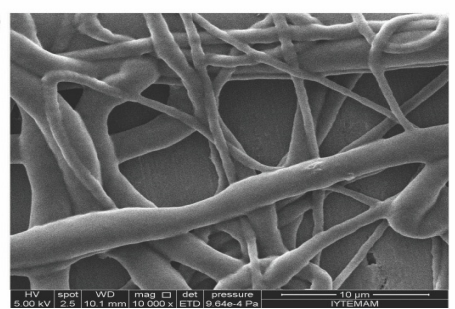




\section{CONCLUSION}

PUs, due to their structure/property diversity, are considered one of the most bio and blood compatible materials known today. Properties like durability, fatigue resistance in tensile, compression or shear, elasticity, compliance, "elastomeric character," and propensity for healing became attainable via PU. The future use of electrospun PU materials in practical applications will require good mechanical properties. Thus, several authors recently investigated the mechanical properties of electrospun PU fiber mats[5].Main reason for choosing PUs in electrospinning is that, the properties of PUs can vary in a wide range, so it is possible to adopt PUs to many applications by varying the structure of PUs. It can also be mixed either with a natural polymer or with a synthetic polymer for special applications such as collagen, dextran, and hydroxypropyl cellulose (HPC). Although there is ever increasing literature on use of PU nanofibers for various applications but still the field is in its infancy. For example, there are also lots of questions for biomedical applications, like interaction of scaffolds with biological systems, toxicity, in vivo studies etc. There is no doubt that PU electrospun materials are going to take major place in future for above mentioned applications. Thus, all these applications have to be thoroughly investigated before the technology can be used for any real practical application.

\section{REFERENCES}

Barick A.K. \& Tripathy D.K. (2010). Thermal and dynamic mechanical characterization of ther- moplastic polyurethane/organoclay nanocomposites prepared by melt compounding. Materials Science and Engineering: A, 527(3), 812-823.

Demir M.M., (2002). Electrospinning of polyurethane fibers. Polymer, 43(11), 3303-3309.

Huang Z.M., Zhang Y.Z., Kotaki M, Ramakrishna S. (2003). A review on polymer nanofibers by electrospinning and their applications in nanocomposites. Composites Science and Technology, 63(15), 2223-2253.

Khil M.S., (2003). Electrospun nanofibrous polyurethane membrane as wound dressing. Journal of Biomedical Materials Research Part B: Applied Biomaterial, 67(2), 675-679. 
Martin, D. J., (2012). Thermoplastic polyurethane (TPU) - based polymer nanocomposites. Advances in Polymer Nanocomposites: Types and applications. Cambridge: Woodhead Publishing Limited, 651.

Mi H-Y,(2013). Characterization and properties of electrospun thermoplastic polyurethane blend fibers: Effect of solution rheological properties on fiber formation. Journal of Materials Research, 28(17), 23392350.

Mondal S. (2014). Influence of solvents properties on morphology of electrospun polyurethane nanofiber mats, Polymers Advanced Technologies, 25:179-183.

Padois K, (2010).Characterization of a polyurethane-based controlled release system for local delivery of chlorhexidine diacetate. European Journal of Pharmaceutics and Biopharmaceutics, 74(2), 255-264.

Ramakrishna S, Fujihara K, Teo W, Lim T, Ma Z. (2005). An Introduction to Electrospinning and Nanofibers. World Scientific Publishing, 285-291.

Sen, R. (2004). Preparation of single-walled carbon nanotube reinforced polystyrene and polyurethane nanofibers and membranes by electrospinning. Nano Letters, 4(3), 459-464.

Yanilmaz M, Kalaoglu F, Karakas H. (2013). Investigation on the effect of process variables on polyurethane nanofibre diameter using a factorial design. FIBRES \& TEXTILES in Eastern Europe, 21, 2(98) 19-21. 